\title{
Desarrollo de dispositivo para determinar las fuerzas en la unión socket-muñón en pacientes con amputación transfemoral
}

\author{
pags 51-65
}

Grupo de Investigación: diseño avanzado

Línea de investigación: Biomecánica

Miguel Moralese, Omar Pérezeo \& Marlon Vargaseo»

\section{RESUMEN}

El presente artículo trata sobre el desarrollo de un dispositivo, para la determinación de fuerzas en la unión socket-muñón, en pacientes reales. El método utilizado fue por medio de una adquisición de datos realizada en 3 pacientes, cada uno con condición de amputación a nivel transfemoral. Se utilizaron sensores Flexiforce, los cuales se distribuyeron en diferentes zonas al interior de la prótesis. Por medio del software Labview y una tarjeta de adquisición de datos Arduino, se realizó una serie de pruebas, durante el ciclo de marcha humana para cada paciente. El resultado se expresa en graficas de fuerza y fase del ciclo de marcha, teniendo en común que la fase, con mayor fuerza registrada, es la fase de apoyo del monopodal realizado por la prótesis.

Palabras claves: adquisición de datos, interfaz socket-muñón, Labview, amputación transfemoral

\section{ABSTRACT}

This article discusses the development of a device for determining forces in the union socket-stump determination of forces in the union socket-stump to real patients; the method used was by a data acquisition performed in 3 patients, each one with a transfemoral amputation condition level, Flexiforce sensors were used, which they were distributed in different areas within the prosthesis. Through the Labview software and card data acquisition Arduino; It was performed for each patient a series of tests during the human gait cycle, which has the graphic result of strength and phase of the gait cycle, having in common that the phase with greater force recorded is the stance phase of monopodal made by the prosthesis.

Keys words: data acquisition, interface socketstump, Labview, transfemoral amputation.

\footnotetext{
- Ingeniero mecánico, especialista en educación y docencia universitaria. Docente investigador y del programa de Ingeniería mecánica de la Fundación Universidad de América. miguel.morales@investigadores.edu.co

- Estudiante coinvestigador del programa de ingeniería mecánica de la Fundación Universidad de América.

•.. Estudiante coinvestigador del programa de ingeniería mecánica de la Fundación Universidad de América.
} 


\section{INTRODUCCIÓN}

La automatización tiene como objetivo mejorar procesos industriales, de manera que evita la interacción humana para ciertos trabajos, comúnmente para el manejo de software y Hardware de adquisición de datos (National Instruments Corporation, 2015). Cuando se habla de procesos industriales, se hace referencia a la intervención de elementos de medición, que indican cómo se encuentra el entorno a trabajar. Este tema hace referencia al procesamiento de datos de una red, entre el sistema de manejo de sensores y la plataforma del software Labview. Los estudios realizados con el método FEA (análisis de elementos finitos), conforman gran parte de las investigaciones acerca de los esfuerzos generados por una persona, en la unión socket-muñón para amputación transfemoral. La tarea de los autores de esta investigación es comparar los datos simulados por el método, con los resultados hechos en esta investigación en pacientes reales.

\section{MATERIALES Y MÉTODOS}

El estudio presente en este documento se basó en el manejo de las plataformas de programación, del software Labview y de las tarjetas Arduino. Otra parte, en los dispositivos de medición de carga (sensores de fuerza). El uso del software de programación LabView, conformó esencialmente la recopilación de datos y gráficas, marcando las señales emitidas por el sensor de fuerza. Para mejor entendimiento, se mostrará los pasos que se tuvieron en cuenta, para la programación de esta plataforma (Diagrama de flujo 1).

\section{Diagrama de flujo 1. Programación de LabView.}

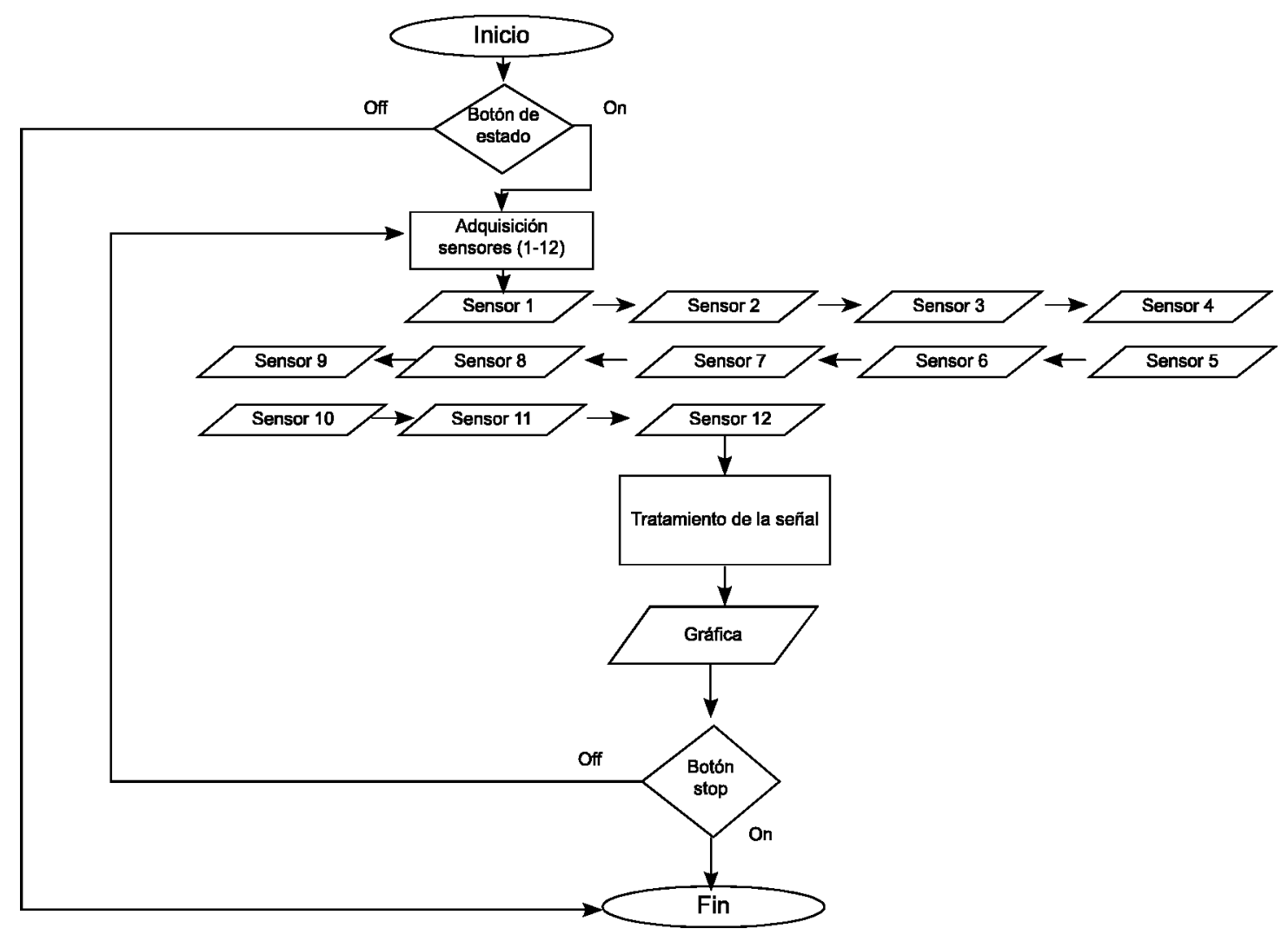

Fuente. Los autores. 
LÍNEA DE INVESTIGACIÓN: BIOMECÁNICA

Tabla 1. Instrumentos virtuales

\begin{tabular}{|l|l|}
\hline \multicolumn{1}{|c|}{ (vi) } & \multicolumn{1}{|c|}{ Función } \\
\hline Case structure & $\begin{array}{l}\text { Crea dos eventos (true and false) en el cual el evento true es el que contiene el resto } \\
\text { del diagram de bloque }\end{array}$ \\
\hline While loop & $\begin{array}{l}\text { Ejecuta el diagrama de bloques de forma continua hasta detenerlo con el botón stop } \\
\text { ubicado en la interfaz del usuario }\end{array}$ \\
\hline Init & $\begin{array}{l}\text { Sirve como interfaz de entrada entre la tarjeta de adquisición arduino con el programa } \\
\text { en labview }\end{array}$ \\
\hline Close & $\begin{array}{l}\text { Sirve como parte final de la interfaz arduino-labview, con la cual se cierra el túnel de } \\
\text { información del while loop }\end{array}$ \\
\hline Analog read pin & $\begin{array}{l}\text { Lee la señal analógica de la tarjeta arduino que corresponde al sensor de fuerza en } \\
\text { forma de voltaje dependiendo del pin que sea seleccionado }\end{array}$ \\
\hline Multiply & $\begin{array}{l}\text { Multiplica la señal obtenida por el Analog read pin y la multiplica por una constante } \\
\text { para convertir el valor de voltaje en fuerza }\end{array}$ \\
\hline Gauge & Indicador de la señal de fuerza \\
\hline Insert into array & $\begin{array}{l}\text { Sirve para procesar los datos en tiempo real por medio del shift register el cual procesa } \\
\text { los datos de la señal ekéctrica de la variable fuerza }\end{array}$ \\
\hline Bundle & $\begin{array}{l}\text { Convierte la señal de fuerza de tres sensores en una señal unificada para poder gra- } \\
\text { ficar }\end{array}$ \\
\hline Waterford charts & Grafica en tiempo real las señales de fuerza de los sensores \\
\hline Shift register & Sirve para poder procesar datos obtenidos dentro del while loop \\
\hline Build array & Sirve para extraer del while loop la información procesada por los Insert into array \\
\hline Write to spreedsheet & $\begin{array}{l}\text { Crea una tabla con los datos obtenidos por el túnel de información, en el cual se mues- } \\
\text { tra todos los valores alcanzados por cada sensor }\end{array}$ \\
\hline $\begin{array}{l}\text { Wait until next ms } \\
\text { multiple }\end{array}$ & Controla la velocidad de adquisición del while loop \\
\hline
\end{tabular}

Fuente: Los Autores

Para el tratamiento de señales emitidas por el sensor, se utilizó una interfaz llamada Arduino, la cual es una plataforma de hardware de código abierto, basada en una placa con entradas y salidas, analógicas y digitales compatible con el programa de LabView. Con estas dos plataformas se generó una comunicación de redes, en la cual se intercambiarían datos del entorno y el ordenador. De manera breve se conocerán los elementos que se utilizaron para la conexión de estas plataformas (ver tabla 1).

Conforme a la necesidad de un manejo cómodo y seguro del estudio, se realizó una interfaz inalámbrica que comunica el ordenador con la plataforma de Arduino. El dispositivo utilizado para la comunicación de redes punto a punto se llama XBee. Es fabricado por la empresa Digi y tiene dos tareas: receptor y emisor, dependiendo de la configuración del mismo. Es decir, si el dispositivo se encuentra conectado al ordenador, éste será el receptor que recibe la información de las señales del sensor, provenientes de otro XBee. Estos últimos se llaman emisor, y se encuentra en contacto por medio de una red punto a punto con el receptor.

Por otra parte, la selección del sensor de fuerza, utilizado en la investigación, debe tener parámetros de capacidad máxima de carga, entre $40 \mathrm{~kg}$ ○ $70 \mathrm{~kg}$, a razón de evitar errores durante la lectura de la prueba. Es en la sensibilidad donde 
se puede afectar su estado inicial, por medio de una magnitud física, entre otros parámetros. Al evaluar las variables mencionadas anteriormente, en 3 tipos de sensores, el indicado para el estudio fue flexiforceA201, un sensor de fuerza resistivo de la empresa Tekscan. De acuerdo con este sensor, el fabricante informó el tipo de circuito que puede ser utilizado, junto con las variables que pueden afectar en el sistema con diferentes resistencias (ver figura 1). Además, la utilización del microchip (MCP 6004), que tiene como propiedad amplificar operaciones, ofrece la entrada y la salida de datos. Este microchip tiene un rango de operación de 1.8 a $6 \mathrm{~V}$. El microchip fue cambiado por una tarjeta de adquisición de Arduino "Arduino Mega", con el fin de permitir una comunicación directa, entre Labview y la tarjeta de adquisición, aumentando la capacidad de conexión de los sensores.

El dispositivo Arduino funciona con una alimentación de $5 v \mathrm{DC}$, permitiendo la conexión de 16 entradas analógicas, que son utilizadas por los sensores. Los sensores varían su resistencia al momento de recibir peso, lo que aumenta o disminuye el voltaje de salida en el Arduino (Ver figura 2).

\section{Figura 1. Circuito Flexiforce}

Luego de obtener el circuito apropiado para el proyecto, el siguiente paso es encontrar el tipo de resistencias (Rf) que se usará en el circuito. Para este caso fue necesario descubrir la resistencia (Rs) y el voltaje de salida (Vt), que emite el sensor al variar su peso. Estas pruebas consistían en el uso de dos instrumentos de medicion, el voltimetro para encontrara la diferencia de potencia, y el ohmimetro que muestra la resistencia electrica (Ver tabla 2). Teniendo en cuenta el voltaje de entrega del Arduino, de 5 voltios, (5Vout), se realizaron pruebas con diferentes cargas, las cuales fueron en aumento, de 5 en 5, hasta 25 lb (Ver tabla 2).

Al tener los datos, obtenidos de las mediciones mencionadas anteriormente, se usó la ecuación entregada por el fabricante (Ecuación 1). Ésta se encontraba en términos de Vout, y se despejó para tenerla en términos de resistencia "Rf" (Ecuación 2 ).

Esta ecuación establece que la resistencia del sensor es igual al voltaje dado por el dispositivo, sobre el voltaje de salida, conformándose el sensor por la resistencia que él emplea, con respecto al peso implementado.

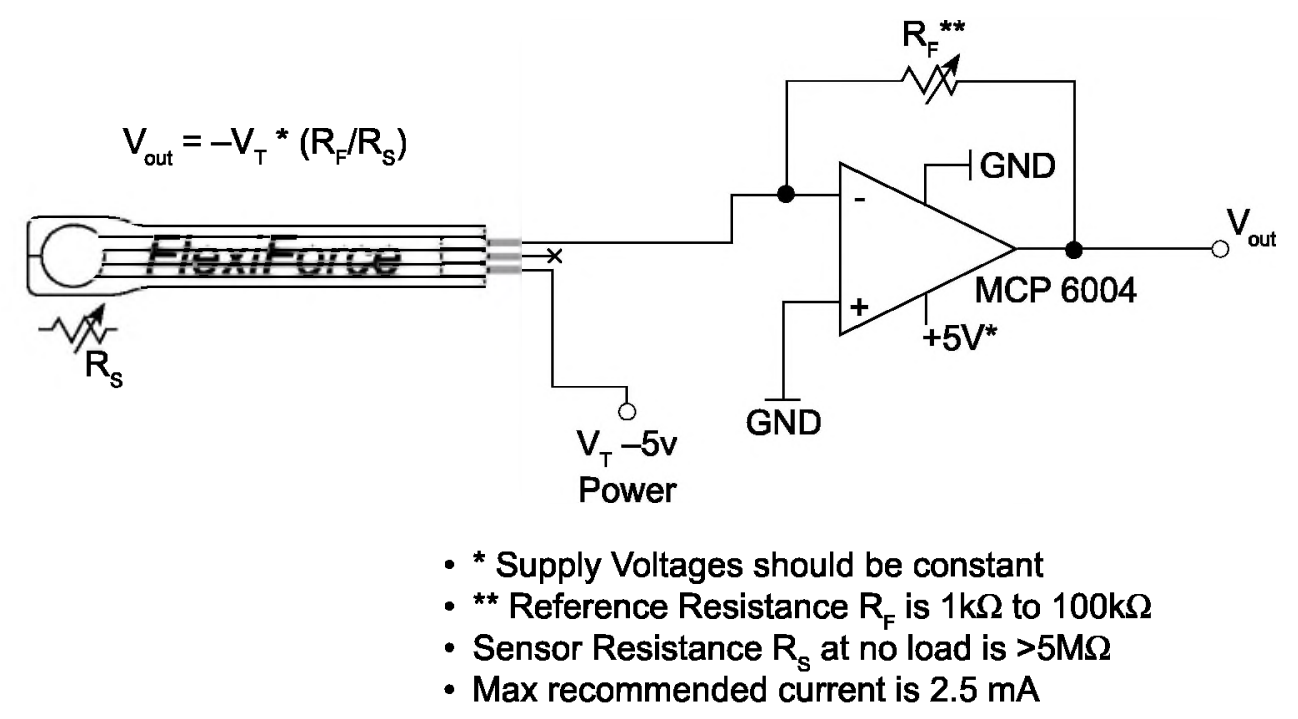

Fuente:TEKSCAN 
LINEA DE INVESTIGACIÓN: BIOMECÁNICA

Figura 2. Circuito Substituido

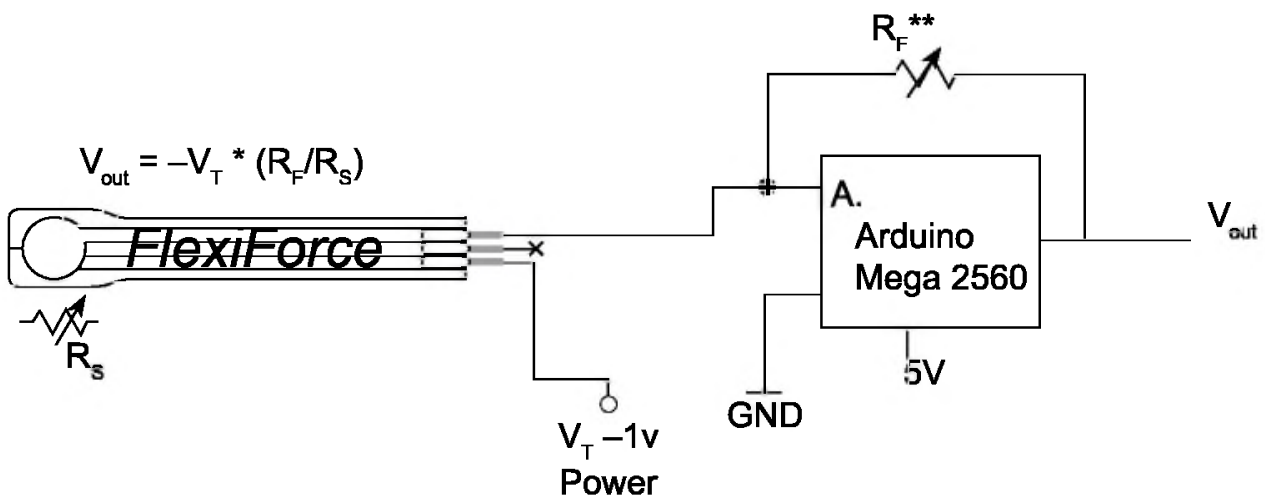

Fuente: Los autores

Ecuación 1. Del fabricante.

Vout $=-(-V t) *\left(\frac{R f}{R s}\right)$
Ecuación 2. Términos en Rf

$R f=\left(\frac{\text { Vout }}{-(V t)}\right) * R s$

Tabla 2. Valores de Rs

\begin{tabular}{|c|c|c|c|c|}
\hline $\begin{array}{c}\text { Vout (V) } \\
5\end{array}$ & $\begin{array}{c}\text { Cargas (Lb) } \\
5\end{array}$ & $\begin{array}{l}\text { Rs (Q) } \\
1791,4 \\
\end{array}$ & $\begin{array}{l}\text { Vt }(\mathbf{V}) \\
0,23 \\
\end{array}$ & $\begin{array}{c}\text { Rf (Q) } \\
38943,48\end{array}$ \\
\hline & 10 & 3056,2 & 0,392 & 38982,14 \\
\hline & 15 & 3742 & 0,48 & 38979,17 \\
\hline & 20 & 5153,2 & 0,661 & 38980,33 \\
\hline & 25 & 5734 & 0,735 & 39006,80 \\
\hline
\end{tabular}

Fuentes: Los autores

Observando los valores entregados por la ecuación para hallar Rf, se llega a la aproximación del valor de $39 \mathrm{~K} \Omega$ de una resistencia estándar. Se genera poco error durante la medición, al comparar con las demás resistencias, se genera una variación de $0.200 \mathrm{~K} \Omega$ a 0.150 $\mathrm{K} \Omega$ dentro del valor de Rf.

Al conocer el tipo de resistencia, manejable en el dispositivo, se dispuso la fabricación del circuito impreso, para conectarse la plataforma
Arduino y realizar las pruebas con el programa de Labview (figura 3). Para esta prueba se fabricó una mesa de carga, que al ponerle un peso, se presionaría el sensor y éste emitiría una señal a la plataforma de LabView. Esto, con la finalidad de conocer las variables que tiene el voltaje a diferentes cargas. La ecuación establece que la resistencia del sensor es igual al voltaje entregado por el dispositivo, sobre el voltaje de salida, por la resistencia que emplea éste con respecto al peso implementado. 
Figura 31. Dispositivo electrónico.

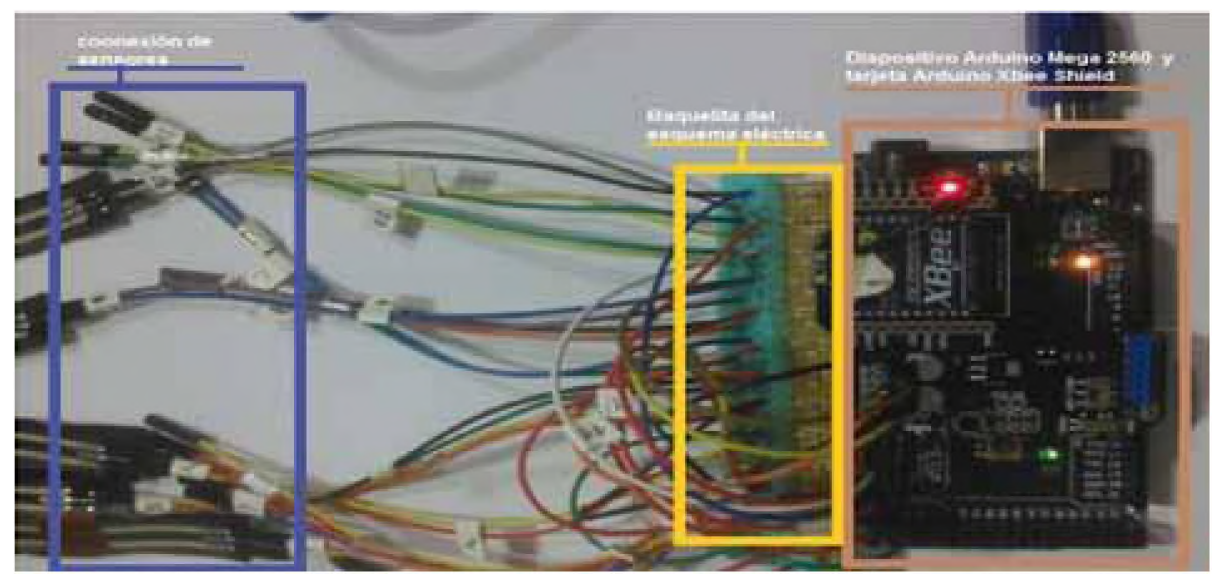

Fuente: Los Autores.

\section{Ecuación 3. Peso en Lb.}

$$
Y=19,266 X^{3}-25,714 X^{2}+26,765 X
$$

Fuentes: Los autores.

Al emplear el sistema internacional en la investigación se realizó una modificación en la ecuación con la finalidad de que los datos entregados fuesen en términos de Newton como se muestra en la ecuación 4.

\section{Ecuación 4. Fuerza en Newton.}

$$
Y=\left(\frac{19,366 X^{3}-25,714 X^{2}+26,765 X}{2,2}\right) * 9,8
$$

Gráfico1.Función polinómica, de 3er. orden.

Carga vs. voltaje

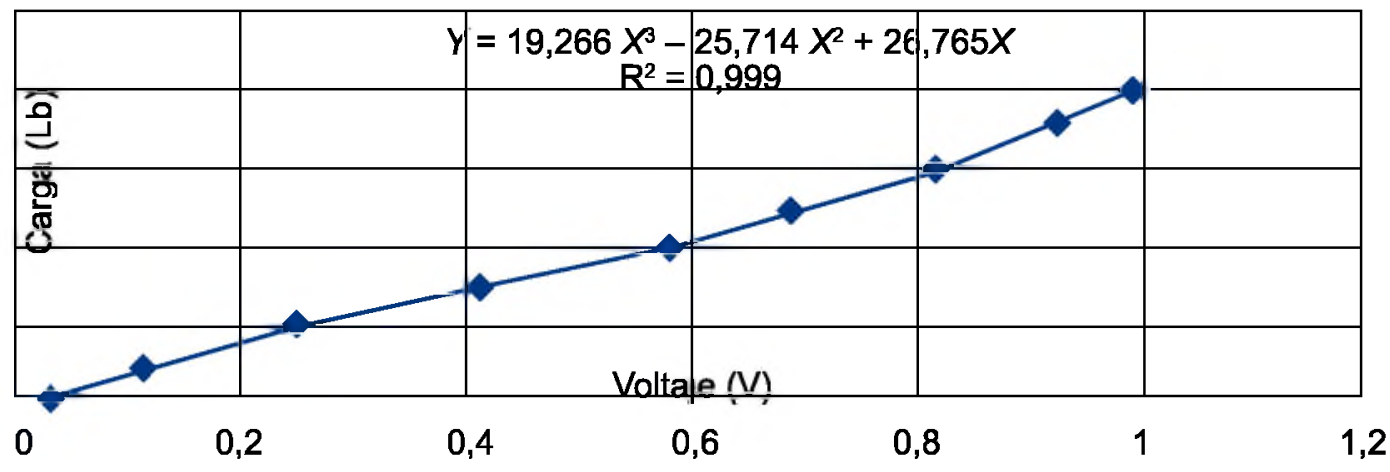

Fuentes: Los autores

Para la medición de los puntos de carga presentes en la unión Socket-muñón, se ubicaron los sensores en grupos de 3, cada grupo distribuido de forma vertical, sobre 3 puntos críticos en la prótesis. Los puntos fueron hallados al dividir el muñón en 5 partes. Se seleccionó de la 2 a la 4, dado que estos puntos eran los tomados como críticos, al momento de realizar la prótesis, por la Fundación Fuente de Esperanza. Cada grupo de sensores se ubicaron en 4 zonas de ésta: frontal, lateral derecha, posterior y lateral izquierda (imagen 4). 
- Estática: Este tipo de evaluación consiste en que el paciente se queda un momento de pie apoyando todo su peso en ambos miembros inferiores, con la finalidad de encontrar los puntos críticos que se tiene en cada uno.

- Ciclo de la marcha humana: A diferencia de la anterior prueba, consiste en que el paciente realice su marcha sin ninguna alteración al caminar. Cada paciente utilizo diferente tipo de apoyo como un bastón, muletas y en el último caso sin ningún tipo de apoyo.

La finalidad de estas pruebas es encontrar las zonas conteniendo una mayor concentración de cargas, en la unión socket-muñón del paciente. El primer paciente evaluado tiene 57 años y cuenta con una amputación transfemoral, en el miembro inferior izquierdo. El tiempo de discapacidad es de aproximadamente 1 año, en el cual nunca ha utilizado una prótesis de tipo transfemoral. Para la ejecución de la prueba se ubicaron los sensores como se muestra en la figura $5, y$ fueron tomados los puntos de mayor relevancia para la distribución de cargas en la prótesis.

\section{Prueba estática, primer paciente}

Los datos obtenidos con el paciente en la prueba estática, muestran una variación durante la misma, como se observa en la gráfica 2 . Su muñón no es uniforme, por lo que las cargas presentes no son equilibradas, como se esperaba de la prótesis. Con estos datos se realizaron gráficas, las cuales muestran la distribución de cargas para las diferentes zonas, pertenecientes a la unión socket-muñón, zona frontal, posterior, lateral derecha y lateral izquierda.

De acuerdo a la distribución de los sensores en del primer paciente, la zona con un comportamiento diferente es la zona posterior, donde se encuentran ubicados los sensores 10,11 y 12. Como se muestra en la gráfica 6 , el sensor de mayor carga es el número 12, el cual se localiza en la zona superior de la amputación, obteniendo como máximo $12.6155 \mathrm{~N}$. Esto, a diferencia de los sensores 10 y 11 , que en este mismo punto logran un valor entre $0.23799 \mathrm{~N} \mathrm{y}$ $1.7629 \mathrm{~N}$ respectivamente. Esta zona no genera una contacto completo con la prótesis, por tal motivo la fuerza es muy baja.

\section{Prueba marcha humana primer paciente}

Con los datos obtenidos en la prueba de marcha humana, se realizó el mismo procedimiento que en la prueba estática, se muestra la distribución de cargas por las diferentes zonas pertenecientes a la unión socket-muñón, zona frontal, posterior, lateral derecha y lateral izquierdo. La zona con mayor alteración se encuentra entre los sensores 4,5 y 6 , pero a diferencia del sensor 5 y 6 , se encuentra una alteración elevada en el sensor 4, como se puede ver en el grafico 3 . Esto se debe a que cuando el miembro inferior amputado hace contacto con el suelo, genera un acercamiento directo entre el hueso femoral y la prótesis, haciendo que las fuerzas se concentren en la parte inferior del muñón.

\section{RESULTADOS}

La adquisición de datos para los 3 pacientes suministrados por parte de la fundación Fuente de Esperanza, se realizó mediante dos pruebas:

En el gráfico, los puntos señalados corresponden a las fases desarrolladas en un ciclo de marcha humana (figura 6), teniendo el número 1. Contacto del talón, 2. Apoyo plantar 3. Apoyo medio, 4. Elevación del talón, y 5 . despliegue del pie (Hernández, 2008).

El segundo paciente evaluado tiene de 26 años. Cuenta con una amputación transfemoral en el miembro inferior derecho. El tiempo de discapacidad es de aproximadamente 1 año, en el cual se ha movilizado con ayuda de muletas. La distribución de los 12 sensores al interior de la prótesis se realizó en las zonas llamadas superior, media e inferior (figura 7), con la finalidad de encontrar alguna alteración de cargas entre el contacto del muñón y la prótesis. 
Figuro 4. Ubicación de dispositivo y sensores.

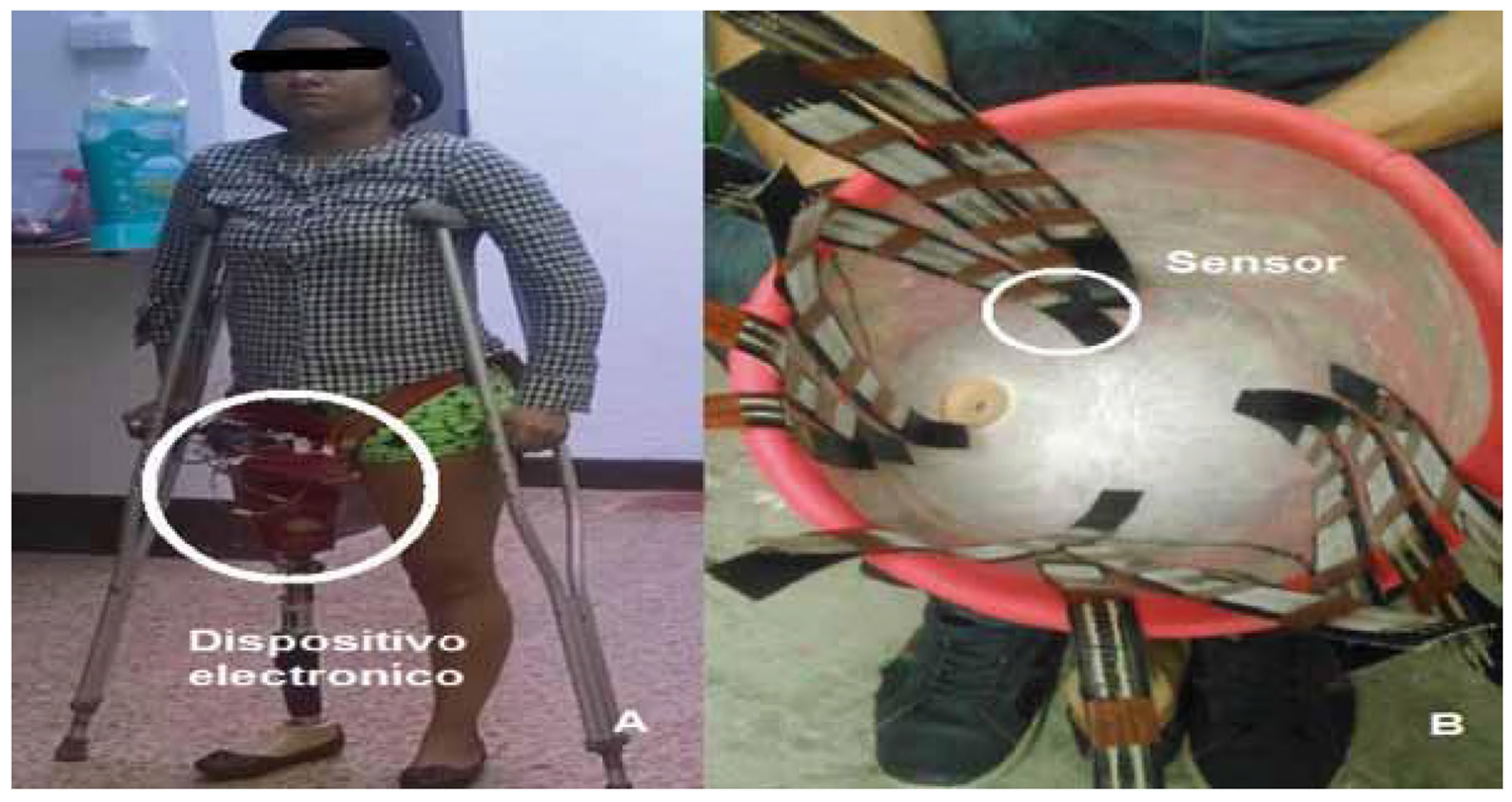

Fuente: Los autores.

A) Dispositivo electronico en el interior de la interfaz socket-muñon; B) Ubicación de sensores.

Figura 5.Distribución sensores. Primer paciente
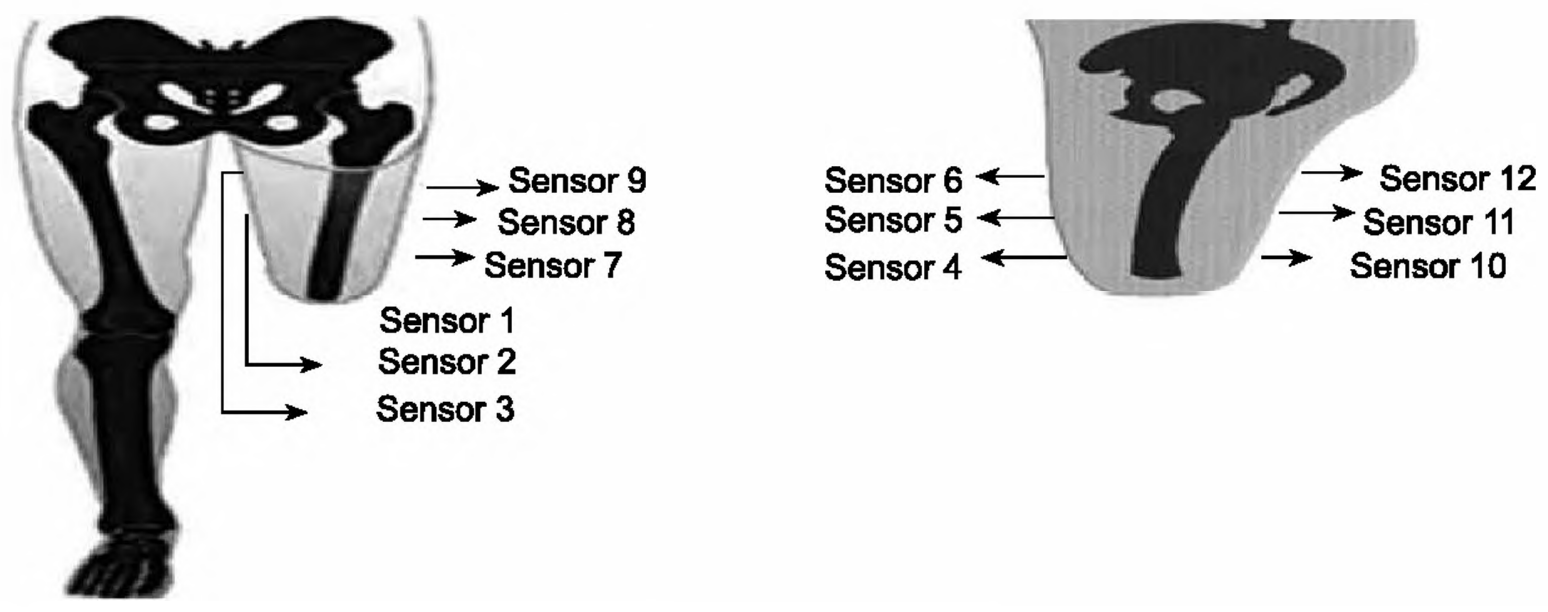

Fuentes: Los autores. 
LÍNEA DE INVESTIGACIÓN: BIOMECÁNICA

Gráfico 2. Zona posterior

\section{Posterior}

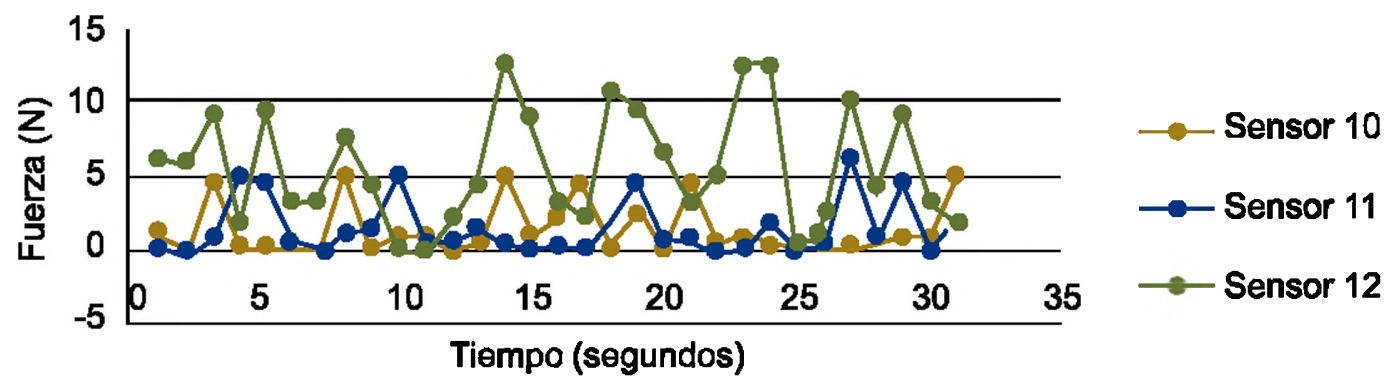

Fuente: Los autores.

\section{Gráfico 3. Zona frontal.}

Frontal

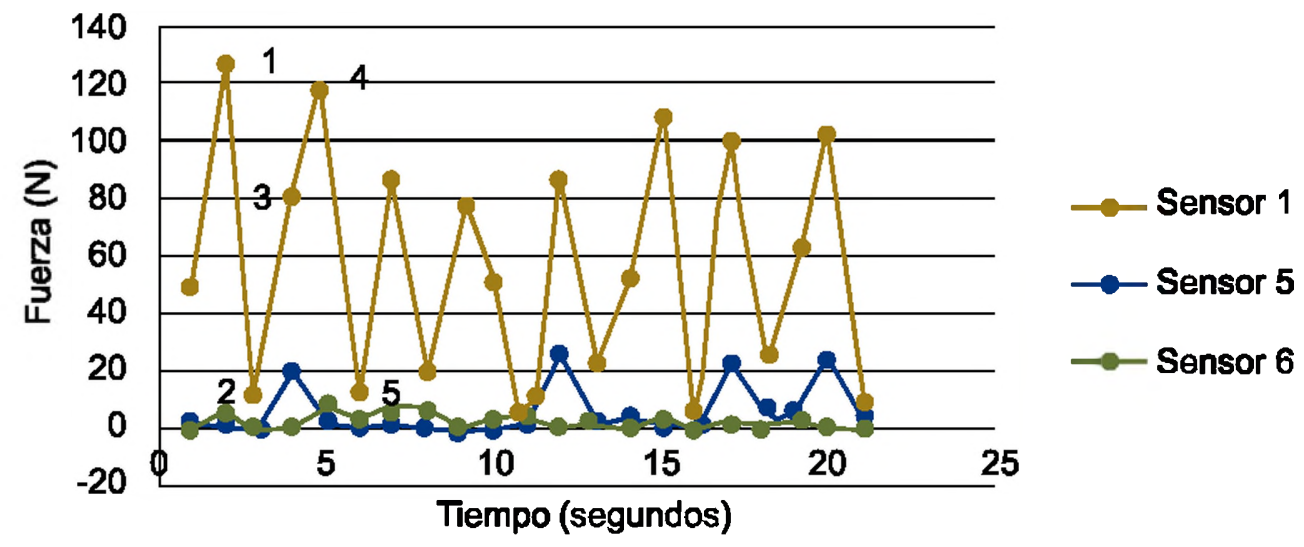

Fuente. Los autores.

Figura 6. Fases de apoyo
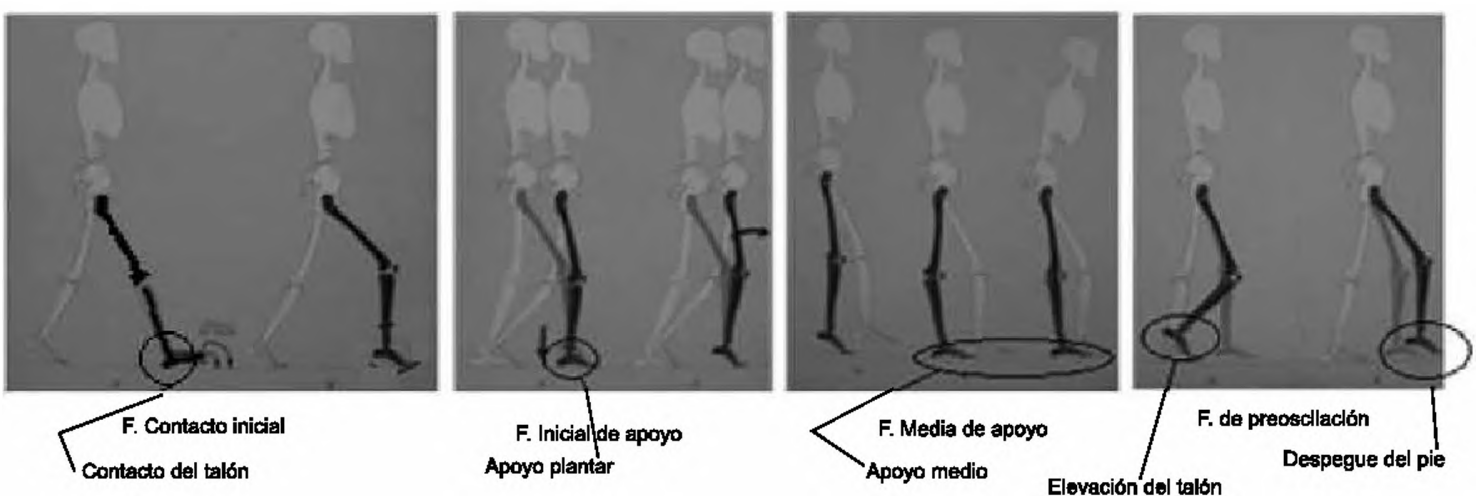

Fuente: Biomecánica de la marcha Patológica de Rodrigo \& Narbón 
Figura 7. Distribución de sensores. Segundo paciente

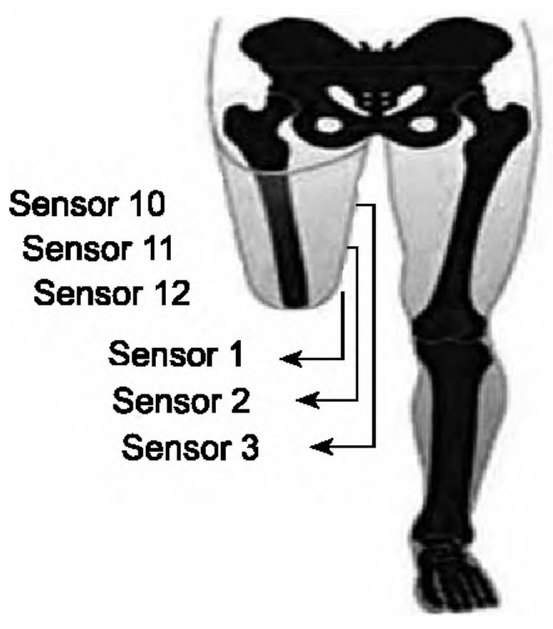

Fuentes: Los autores.

\section{Prueba estática, segundo paciente}

Al observar las gráficas de desempeño para esta prueba estática se encontraron intervalos de equilibrio entre las diferentes zonas censa- das. Pero en el caso del sensor 12 (gráfico 6), que se encuentra localizado en el lateral derecho de la zona inferior de la prótesis, se logra observar una leve elevación con respecto a los sensores 10 y 11 .

Gráfico 6. Zona lateral derecho.

Lateral derecho

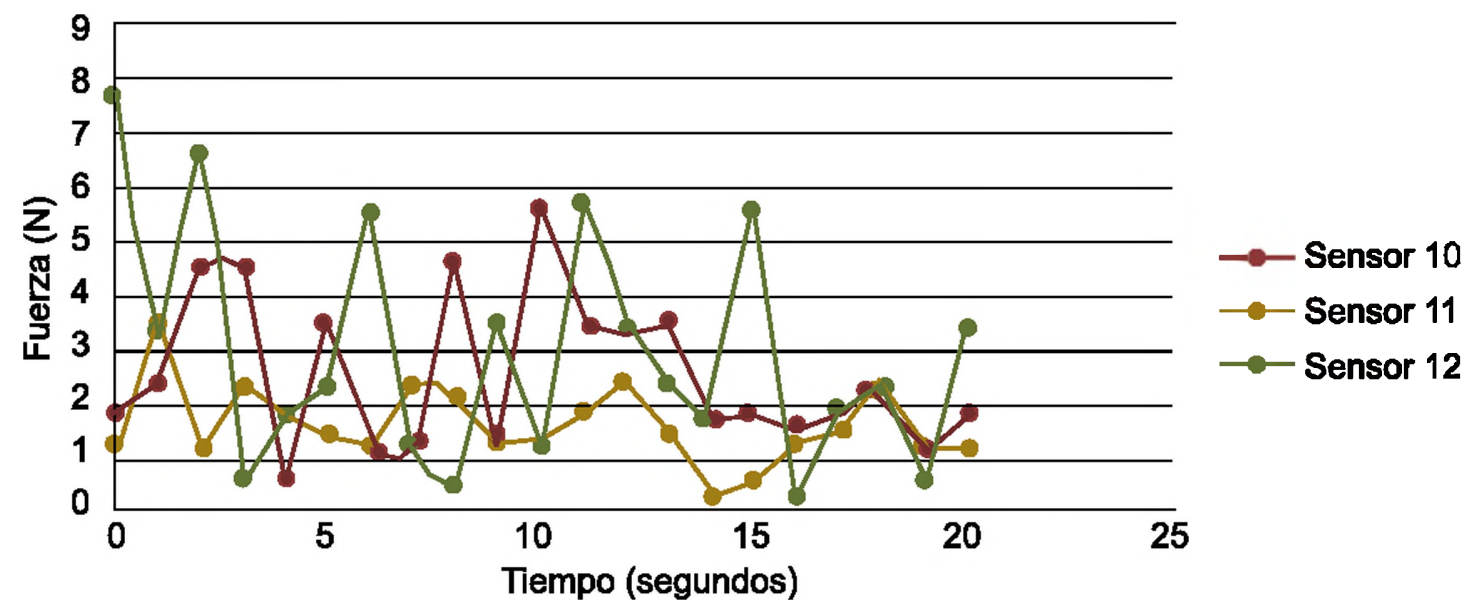

Fuente: Los autores 


\section{Prueba marcha humana segundo paciente}

La prueba de marcha humana con el paciente 2 se consigna una variación entre $0.119 \mathrm{~N}$ a
$5.57 \mathrm{~N}$, este tipo de diferencia está de acuerdo con la fase empleada por la paciente. Se puede observar en el gráfico 7, donde se muestra los datos emitidos por el sensor en la zona frontal de la amputación.

Gráfico 7. Zona lateral izquierda.

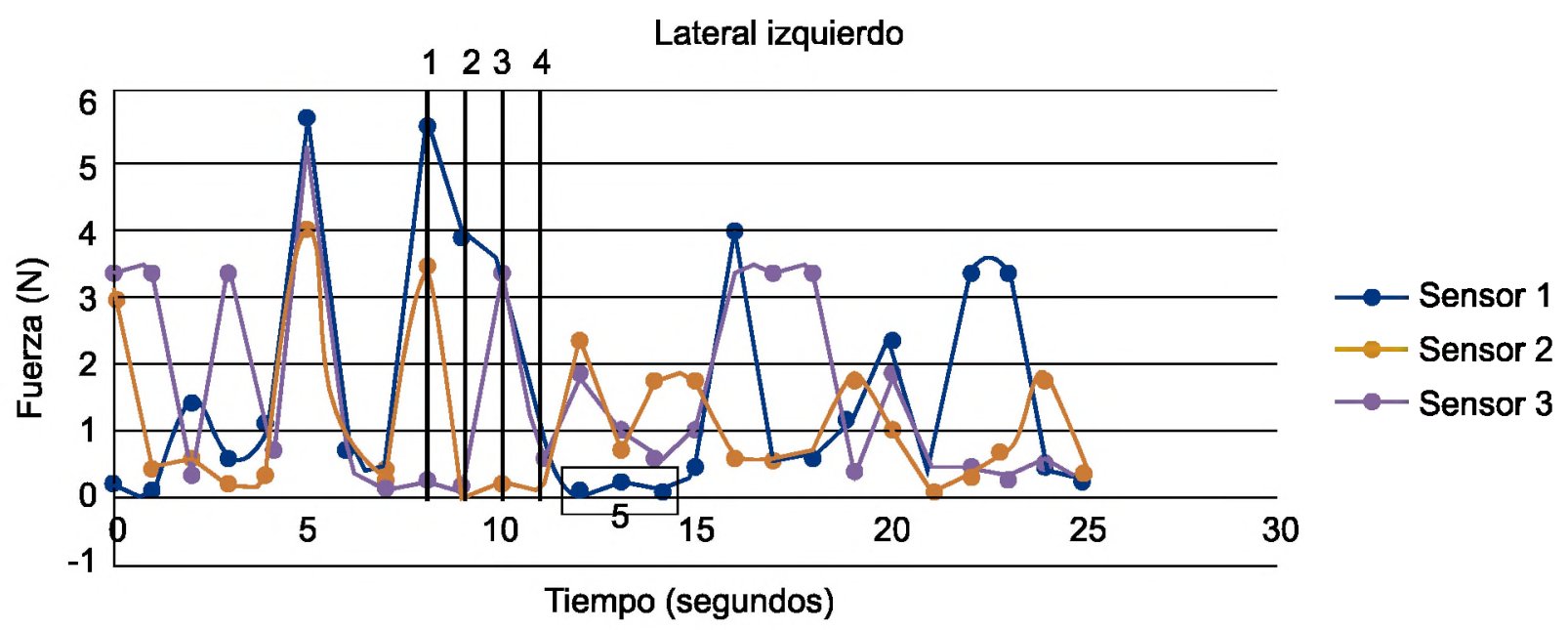

Fuente. Autores.

La zona con una leve afectación de carga, en la prótesis, se encuentra ubicada en los sensores 1,2 y 3 . Es decir, la zona lateral izquierda, a diferencia de las demás zonas con bajos valores, será descrita por su condición. El sensor 1, ubicado en la zona superior del lateral izquierdo, tiene una actividad inusual debido a la marcha presentada por el paciente. Llegó a una fuerza de $5.577 \mathrm{~N}$, en el contacto del talón con el suelo. Esta alteración es debida a que, al momento de realizar la marcha, el movimiento de la cadera es exagerado y elevado, al mover la pierna con la amputación. Es decir, el muñón tiende a salirse del eje sagital.

El paciente evaluado no. 3, tiene una edad de 39 años. Cuenta con una amputación transfemoral en el miembro inferior derecho. El tiempo de discapacidad es de aproximadamente 5 meses, durante el cual se ha dedicado a recu- perarse de la amputación, realizando terapias y ejercicio al muñón.

Como se realizó con los anteriores pacientes, la distribución de carga para el paciente, se hizo tomando los tres puntos en cada zona destacada, como se puede ver en la figura 8.

\section{Prueba estática, tercer paciente}

El paciente presentó un equilibrio, en la fuerza existente en el contacto del muñón y la prótesis, como se puede observar en la tabla 14. Los datos mantienen un rango de $3.245 \mathrm{~N}$ a $5.013 \mathrm{~N}$, localizadas en la zona censada. Se observaron las diferentes señales emitidas por cada sensor, y se nota una diferencia entre la zona lateral derecha, en el sensor 7 , donde se encuentra un bajo contacto del muñón con la prótesis (grafico 8). 
Figura 8. Distribución de sensores tercer paciente
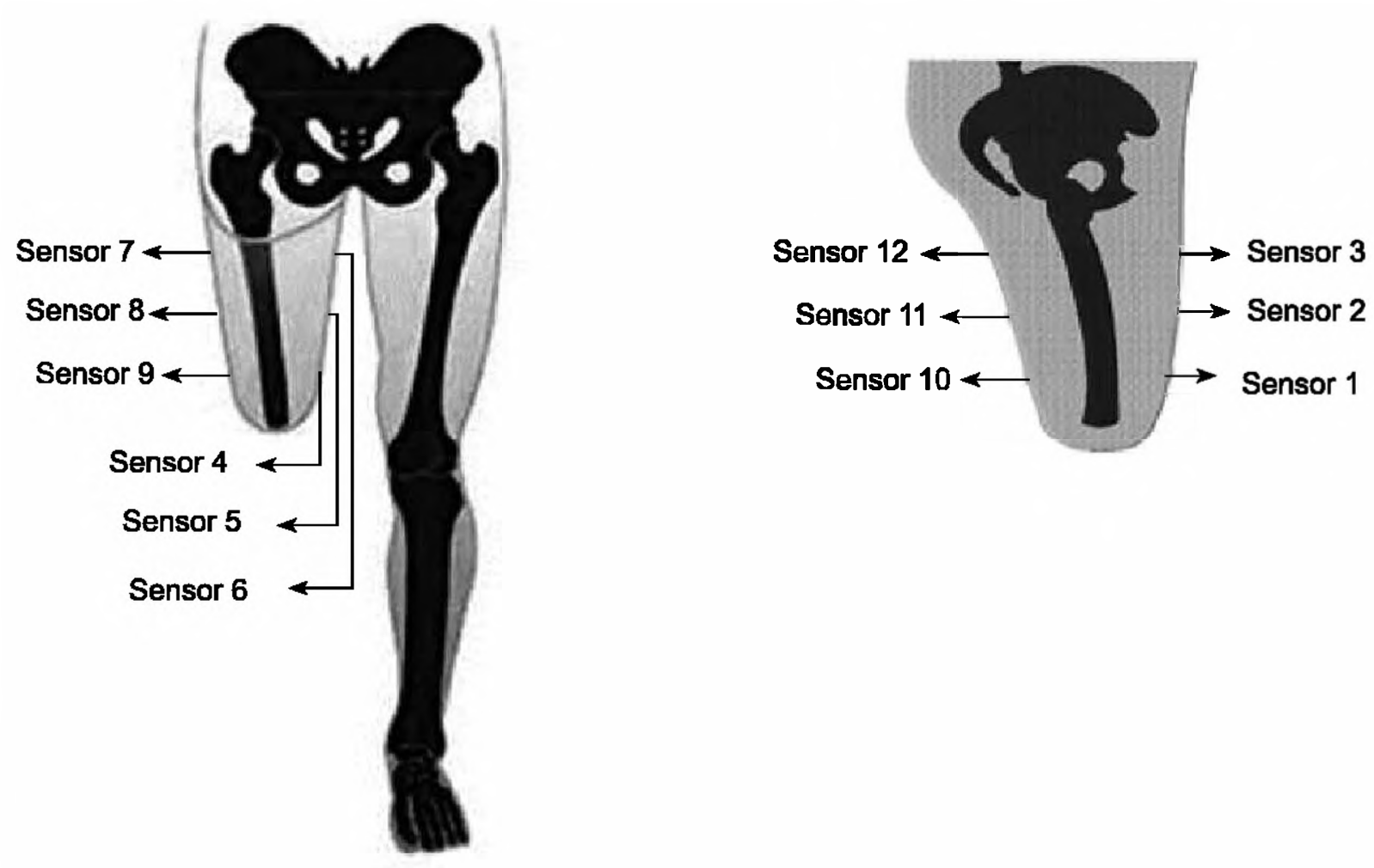

Fuente. Los autores

Gráfico 8. Prueba estatica

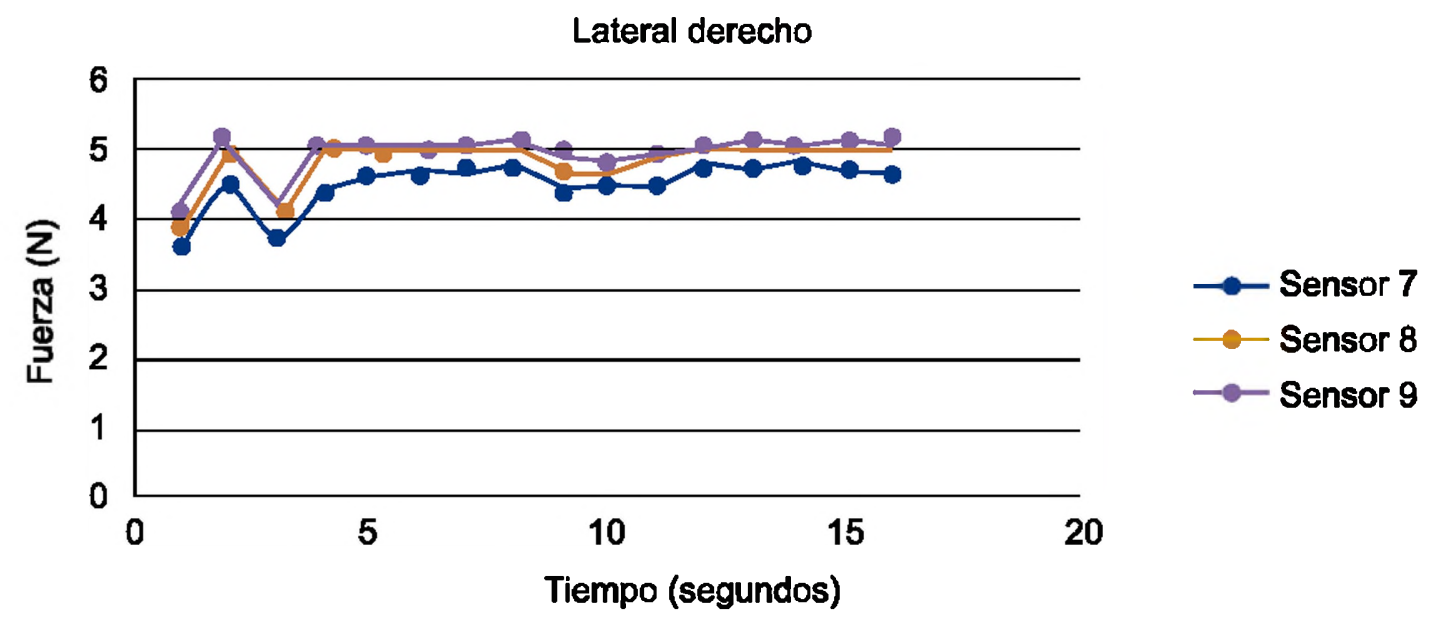

Fuente. Los autores 
Gráfico 9. Lateral izquierdo

Lateral izquierdo

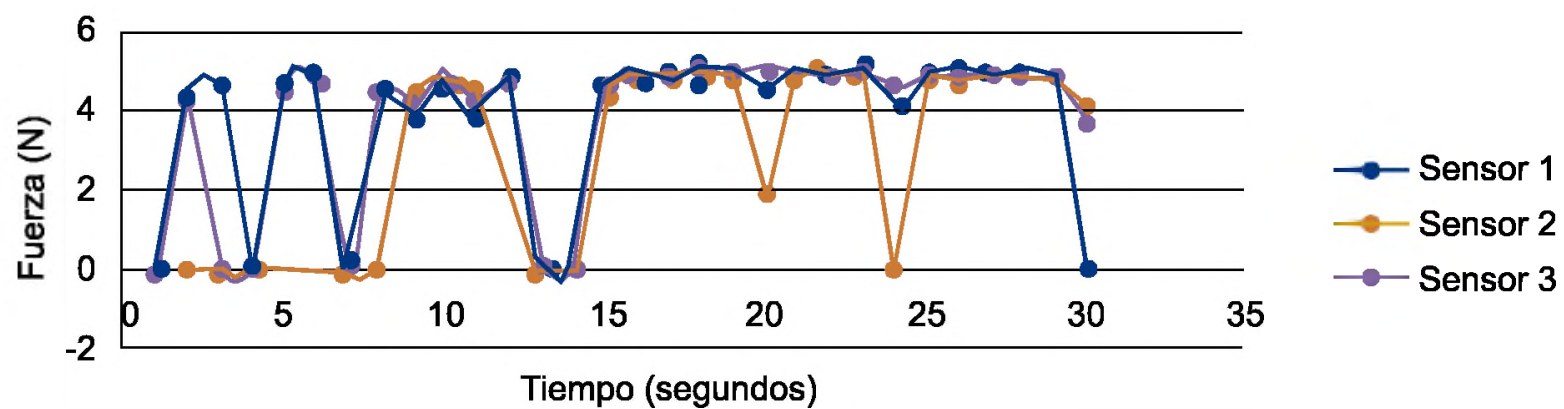

Fuente. Los autores

\section{Prueba marcha humana tercer paciente}

Para la prueba de marcha humana, los datos obtenidos de los sensores 1,2 y 3 , muestran una señal muy similar para cada punto, generada durante la marcha misma. Esto se debe a que la prótesis estaba ajustaba al muñón, y se encontraban las variaciones para cada fase de la marcha (grafico 9). Sin importar el tipo de corte realizado en el muñón, las cargas se comportaron de una manera distributiva.

Comparado con la prueba estática, aunque la distribución de cargas aumentó, se mantuvo el mismo comportamiento de la distribución. La zona de mayor carga en la unión socket-muñón, la cual se encuentra en la parte frontal, con un valor máximos de $5,013 \mathrm{~N}$, está en el sensor 3 , 10 y 11.

\section{DISCUSION}

Los resultados obtenidos en esta investigación, se compararon con resultados provenientes de la literatura donde, según la tesis titulada "Nivel de confort y distribución de esfuerzos en la Interfaz Socket Muñón en amputados transfemorales." (Ramírez, J. 2011); los valores máximos de presión se generan en la etapa de bipedestación, con un valor de $1,7 \times 10^{5} \mathrm{~Pa}$. Esta presión es ejercida en la zona superior, y parte posterior de la prótesis transfemoral (figura 9). Estos datos fueron obtenidos por medio de la simulación de la interfaz socket-muñón, en un programa FEM. Comparada con estos valores, la etapa de bipedestación hace referencia a la prueba estática realizada, en la cual los valores máximos se encuentran en $2,08 \times 10^{5} \mathrm{~Pa}$. Este está ubicado en la parte posterior de la zona superior de la interfaz socket-muñón.

En el artículo titulado: "Design process of a mechatronic device for measuring the stump stresses on a lower limb amputee." (Áviles, Hernández, Jiménez \& Caldas, 2013), se desarrolló un método para mediciones experiméntales de esfuerzos. Éste se basó en crear un muñón a base de gel balístico, en el cual se colocaron grupos de sensores para medir los esfuerzos sobre la interfaz socket-muñón, simulando condiciones de marcha humana. Teniendo como resultado una presión máxima en la parte inferior de la prótesis, de 2,56 Mpa, comparado con el con el primer paciente evaluado con 1,22 Mpa, se ubicó en la parte frontal del zona inferior de la interfaz

\section{CONCLUSIONES}

El sistema de comunicación inalámbrica, punto a punto, brindada por el Xbee, facilita la comunicación entre ordenador y el dispositivo. Se emiten las señales de los sensores, sin ge- 
nerar ningún tipo de intervención, permitiendo al paciente realizar la marcha normalmente. Se obtienen así los datos precisos y claros de lectura, acerca de las fuerzas encontradas en el interfaz socket-muñón.

La tarjeta de adquisición Arduino suministra los valores obtenidos por los sensores, en términos de voltaje, contando con los instrumentos virtuales presentes en el software Labview. Se convirtieron los datos de voltaje a fuerza, con el fin de analizar los términos en tiempo real, mientras el paciente realiza la marcha.

Figura 9. Estado de esfuerzos muñón.
El dispositivo de medición desarrollado en esta investigación es versátil, debido a que cada sensor puede ser ubicado en diferentes posiciones, según la aplicación lo requiera. Se permite la utilización del dispositivo para diferentes investigaciones futuras, como el caso de cualquier amputación del tren inferior.

Al comparar los resultados obtenidos con otras investigaciones, se encuentra que los valores realizados, bajo diferentes modelos de simulación en programas FEM, concuerdan con los resultados de esta investigación, validando así la determinación de las fuerzas en la interfaz socket-muñón.
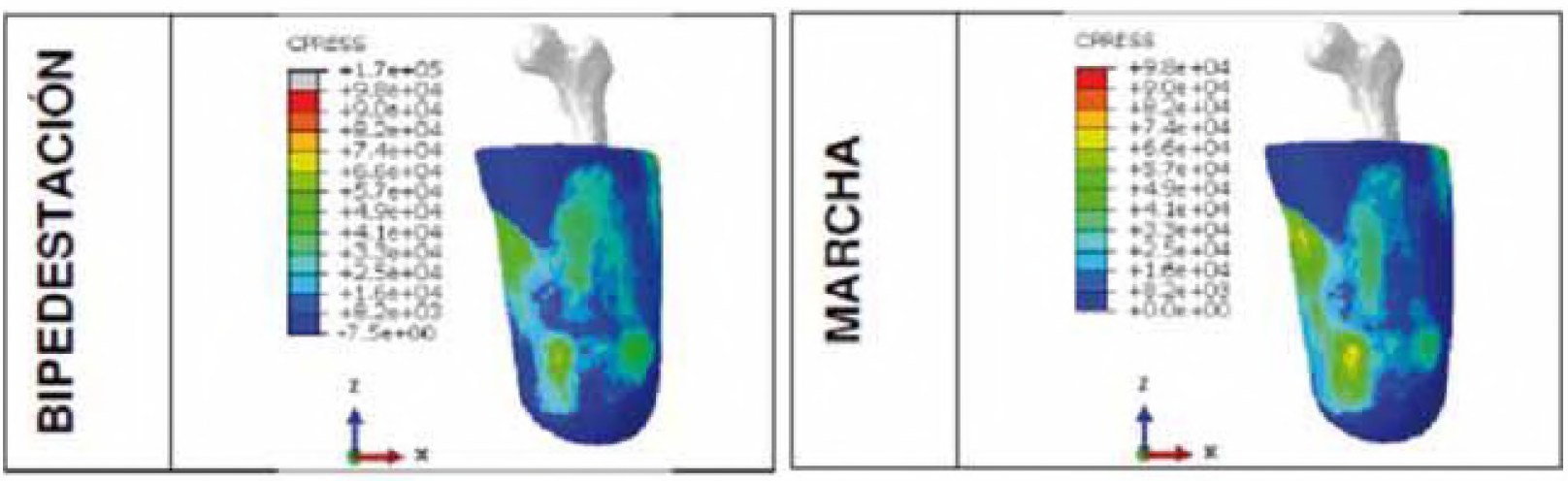

Fuente: Ramírez, J. (2011) “Nivel de confort y distribución de esfuerzos en la interfaz socket - muñón en amputados transfemorales.' Tesis. Medellín, Universidad Nacional de Colombia. Facultad de minas.

\section{REFERENCIAS}

Universidad de las Américas de puebla. (2011). "Galgas extensométricas: sus tipos y principios". Cholula, puebla : facultad de ingeniería. N.e.

Arduino verkstad ab. (2013). "Qué es arduino". Sitio web de creative technologies madrid. [en línea] fundación telefónica, 14 de marzo. [citado el: marzo de 2 de 2014.] Http:// madrid.verkstad.cc/es/courseliterature/que-es-arduino/. N.e.

Aula virtual (2010). "Método del puente de wheatstone. Medicion de resistencia por el metodo del puente de wheatstone". N.e : exactas.dyndns. org. N.e.

Áviles, O., Hernández, J., Jiménez, S. \& Caldas, O.
(2013) “Design process of a mechatronic device for measuring the stump stresses on a lower limb amputee". Articulo. Bogotá: Universidad Militar Nueva Granada.

Cyclopaedia.net.

(2009)

"Transistor unión". Electrónica y microelectrónica para científicos. N.e : cyclopaedia.net. Aula virtual. N.e. 
Departamento de ingenieria electrica y de computadoras. (2010) "Transductores". Buenos aires, Universidad Nacional del Sur, (cod.2774).

Escuela de ingeniería eléctrica. (2013) "Transductores y sensores". El salvador, Universidad del Salvador. N.e.

Fundación fuente de esperanza. fundafe colombia . 2015. Hemos encontrado en ayudar la mejor manera de vivir. sitio web de Fundación Fuente de Esperanza. [En línea] Fundación Fuente de Esperanza, 22 de enero de 2015. [Citado el: 20 de febrero de 2015.] http:// www. fundafecolombia.org/. n.e.

Hernández stengele, francisco. 2008. Diseño y construcción de prototipo neumático de prótesis de pierna humana. Tesis de grado. Puebla : univer- sidad de las américas puebla, 2008. N.e.

National Instruments Corporation. (2015). "¿Qué es adquisición de datos?" Dallas. 01 8005181773 .

-. (2015) "Software de desarrollo de sistemas ni. Labview". Dallas, National Instruments Corporation. 01800 5181773 .

—. (2015) “¿Qué es automatización?" Sitio web de automatización. [en línea], [citado el: 04 de mayo 15] http://www. ni.com/data-acquisition/pc-based-automation/esa/. N.e.

Ramírez, J. (2011) "Nivel de confort y distribución de esfuerzos en la interfaz socket - muñón en amputados transfemorales". Tesis. Medellín, Universidad Nacional de Colombia. Facultad de minas. N.e.
Sosa, J. (2014). "Galgas extensiométricas”. Buenos aires, Universidad Nacional de la Plata. Facultad de ingeniería. N.e.

Transductores-instrumento. 2010. Piezoeléctrico. Sitio web de transductores-instrumento. [En línea] Blog de pdf. [Citado el: 4 junio del 2014.] http:// www.ing.unlp.edu.ar/electrotecnia/tcieye/Apuntes/Transductores\%20_\%20Rev2010. pdf/ n.e

Vera luna, Pedro. 2006. Biomecánica de la marcha humana normal y patológica. Valencia : Paidos, 2006. n.a.

Zepeda, R. (2008) "Control de temperatura en procesos industriales por medio de puerto rs-232 e interfaz labview". Tesis de grado. Azcapotzalco, instituto técnico central. Escuela superior de ingeniería mecánica y eléctrica. At-092/2008. 